\title{
Observing Ethical Principles In Business: A Competitive Advantage?
}

Jana Markesová (E-mail: atomo@atomo.cz), Technical University of Brno, Czech Republic Mirko Dohnal (E-mail: dohnal@fbm.vutbr.cz), Technical University of Brno, Czech Republic Anna Putnová (E-mail: putnova@ fbm.vutbr.cz), Technical University of Brno, Czech Republic

\section{Introduction}

There is an unwritten assumption that success and business ethics are closely interrelated, and that it is not possible, in a successful business, to disregard moral and ethical principles on the long run. Unfortunately, it is extremely difficult to verify such an assumption.

Knowledge of ethics and its relations with economics are vague, inconsistent, partially subjective and sparse. That is why it cannot be studied using traditional mathematical and statistical methods.

One of the classical methods that can be used to describe uncertainty is the theory of probability. However, the problem of quantifying the probability of an occurrence of a phenomenon lies in its repetition: some situations do not repeat, and a certain object can be under certain conditions studies only once. In areas like economics, management, marketing, investments etc., it is not possible to carry out experiments. There can only be made observations and descriptions of the reality. This is the case also with business ethics and its influence on financial performance of companies.

Such phenomena under study are quite particular, which makes the application of statistics very difficult, if not impossible, and little reliable. What is more, some data in company descriptions and characteristics may be missing, as it was not possible to obtain it. This is why it is not convenient to apply traditional methods to formalize such unique phenomena, relations and dependencies which characterize ethical behaviour of companies and their financial performance.

As a suitable data and knowledge mining calculus to integrate this type of knowledge, some expert systems could be used. Expert systems represent an algorithm based on some kind of fuzzy logic. To apply fuzzy logic, which can be used successfully to solve a broad spectrum of problems, it is necessary to formalize the obtained knowledge and then input it into expert systems.

A research among small and medium enterprises in the Czech Republic (Moravian Region) and Italy (Regions of Emilia Romagna and Lombardia) from the sectors of machine industry and electrotechnics has been carried out in 2002. This research concentrated on the present state of business ethics and its correlation with the economic performance of the companies. The data obtained was interpreted as a fuzzy knowledge base which can be used to evaluate and predict the financial performance of companies on the basis of non-financial factors.

\section{The Fuzzy Set Theory - Tutorial}

The theory of fuzzy sets is based on the premise that the key elements in human thinking are not numbers but words. The most important feature of human thinking is the not yet well-understood ability to extract from a collection of masses of data only such items of knowledge which are relevant to the task at hand. The theory of fuzzy sets allows the existence of a type of uncertainty due to vagueness or fuzziness rather than due to randomness alone. In its most basic sense, a fuzzy set is a set where objects have gradual rather than abrupt transition from membership to non-membership. 
A linguistic value is a "value" that is given by words, e.g. high, low. The variable Management Qualification (QUA), chosen by us among others to describe the present state of Czech SMEs, could be described by the following set of values (e.g. dictionary):

low, medium, high

Each verbal value is then transformed into a fuzzy set by the grade-of-membership function given in Figure 1; it is necessary to specify the grade of memberhip of all sets. The numerical values corresponding to points $a, b, c, d$, are established by a team of experts.

For example, a typical medium (M) QUA is:

$$
\mathrm{b}<\text { QUA }<\mathrm{c}
$$

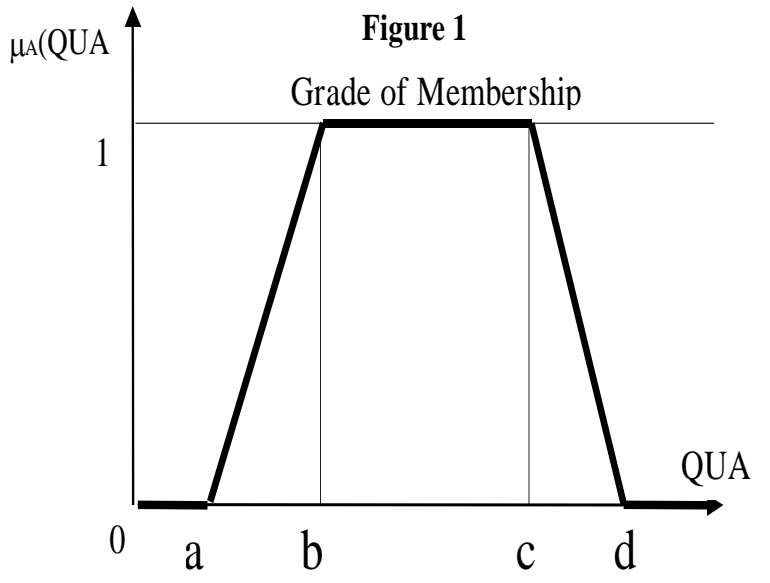

The grade of membership of QUA to the fuzzy set $\mathbf{M}$ is:

$$
\mu_{\mathrm{M}}(\mathrm{QUA}) ; \mathrm{QUA} \in \mathrm{U}
$$

where $\mathbf{U}$ is an universe of variable QUA. The values of QUA described in (1) belong to the fuzzy set medium QUA fully, e.g. with the grade of membership equal to 1 . Therefore they are typical medium QUAs.

Then there are two fuzzy intervals, namely:

$$
\mathrm{a}<\text { QUA }<\text { b; c }<\text { QUA }<\text { d. }
$$

These intervals represent QUA numerical values which belong to the fuzzy set medium $Q U A$ partially.

\section{Fuzzy Models}

A fuzzy model is a set of conditional statements:

$$
\begin{aligned}
& \text { if } A_{1,1} \text { and ..........and } A_{1, n} \text { then } B_{1} \text { or } \\
& \text { if } A_{2,1} \text { and .........and } A_{2, n} \text { then } B_{2} \text { or } \\
& \ldots \ldots \ldots \ldots \ldots . \\
& \text { if } A_{m, 1} \text { and } \ldots \ldots \text { and } A_{m, n} \text { then } B_{m}
\end{aligned}
$$

where fuzzy sets

$$
A_{i, j}, B_{i} ; i=1,2, \ldots \quad m, j=1,2, \ldots n
$$

are one-dimensional fuzzy sets and can be easily specified or/and modified using points $a, b, c, d$ (see Figure 1) for each set.

There are many different fuzzy reasoning algorithms. However, our experience showed that the most important feature of an algorithm is its transparency and simplicity. Methods of knowledge engineering are used to develop the best possible fuzzy models. Some simple algorithms are described in Dubois [3]. 


\section{Creation Of An Argument-Specific Fuzzy Knowledge Base}

\subsection{Definition Of Variables $Y, X_{1}, X_{2}, \ldots \ldots \ldots \ldots . . ., X_{n}$}

With reference to our problematics, e.g. the influence of ethical behaviour on companies' financial performance, the following variables were identified: Management qualification, management knowledge, management changes, management age, management share of property, market share, product/service quality, number of employees, current ratio, total debt, profit in next worth, payment discipline, conjuncture stage, competition, economic influences, protection of environment, index of bankruptcy, adherence to commitments, unfair competition, collaboration of owners and management, discrimination of employees in the workplace, transparency of management decisions, ethical code, index of ethical behaviour.

\subsection{Definition Of A Dictionary For Each Variable.}

Every variable has to be characterized by a set of verbal values (e.g. dictionary). As an example, two dictionaries will be given:
Variable
Verbal description
QUA
management qualification
- $\quad$ low (basic or no education - unsatisfactory)
- medium (professional course, professional school with school-leaving exam, university degree in another field of activity - required)
- $\quad$ high (university degree in the same field of activity, specialized courses studied abroad - more than required)
EI
$\underline{\text { economic influences }}$
- low (low sensitivity of the branch to economic influences, economic influences do not represent a limitation to enterpreneurship)
- $\quad$ medium (economic influences limitate the enterpreneurship partially)
- $\quad$ high (substantial sensitivity of the branch to economic influences, for example the limitation of the enterpreneurship by tax burden)

\subsection{Definition Of A Fuzzy Set For Every Value From Each Dictionary}

Each verbal quantifier is attributed an interval of numerical values (e.g. a fuzzy set). This is done by a team of experts. As an example, the quantification of $Q U A$-values will be shown:

\begin{tabular}{|l|l|l|l|}
\hline $\begin{array}{l}\text { Numerical } \\
\text { Interpretation }\end{array}$ & $\mathbf{a}$ & $\mathbf{b}=\mathbf{c}$ & $\mathbf{d}$ \\
\hline low & 0,0 & 0,1 & 15,0 \\
\hline medium & 10,0 & 50,0 & 90,0 \\
\hline high & 80,0 & 100,0 & 120,0 \\
\hline
\end{tabular}

\subsection{Definition Of A Set Of Propositions With The Use Of Fuzzy Values From The Dictionaries.}

In this case, a proposition is the characterization of one particular company with the help of all the variables mentioned in 4.1. A set of propositions, e.g. the characterizations of a great number of companies, represents the fuzzy knowledge base. In this case, we have accumulated the descriptions of 75 companies (e.g. 75 propositions).

\subsection{Definition Of Weights Of Variables And Propositions.}

As the importance of each variable for the prediction of financial performance and the reliability of every proposition is different, it is necessery to attribute weigts to variables and to propositions. 


\subsection{Testing Of The Fuzzy Knowledge Base.}

Before the fuzzy model can be used practically, it is necessary to test the consistency of the fuzzy knowledge base, e.g. evaluate to which extent the contained data is compact and whether the fuzzy model will have a sufficient reliability. During the consistency test, every single proposition is being checked for consistency with the rest of the fuzzy model. If there is a great number of similar propositions, it is a very positive fact for further real applications of the model. The expert system will not deduce the answer on the basis of just one proposition, but on the basis of several similar propositions.

\subsection{If The Testing Is Not Successful, The Procedure (Starting From 4.1) Has To Be Repeated.}

Experience and engineering "feelings" of different experts are mutually inconsistent. The consequence is that a good fuzzy model can only be developed as a result of a trial-and-error procedure to achieve a generally acceptable compromise within a group of experts. It is the effectiveness with which uncertain knowledge is used, which is very often the main distinction between good and bad models of the same state or process.

In this case, the outcome of the consistency test was positive, so that the developed fuzzy model can then be used successfully to predict for example the financial performance of a company on the basis of other known variables.

\section{The Findings}

After the consistency test, a comparison of the indicator of bankruptcy (IB) with the indicator of ethical behaviour (ETI) was made to find out whether there is some kind of correlation between them.

Presupposition: "A company that disregards ethical principles, will show worse economic performance; in other words: there is a positive correlation between a susceptibility to bankruptcy and a lack of ethical behaviour."

The criteria for judging whether the above presupposition is true, were set as follows:
If $I B=$ high and $E T I=l o w$, the above presuposition is true, since: unethical behaviour influences bad economic performance of a company; if the company disregards moral principles, it can become one of the reasons for bankruptcy.

b) If $I B=$ med and $E T I=$ med, the above presuposition is true, since: mediocre ethical behaviour brings about only mediocre financial results.

c) If $I B=$ low and $E T I=$ high, the above presuposition is true, since: if a company acts in an ethical way, this has a positiv influence on its economic performance; the company's susceptibility for bankruptcy is rather low.

It was found out that the above presupposition was supported by $64 \%$ of propositions relative to Czech companies, and by $77 \%$ of propositions relative to Italian companies. That means that in $64 \%$ of Czech SMEs and $77 \%$ of Italian SMEs there is a direct correlation between the companies' ethical behaviour and their economic performance. The development of business ethics in the Czech Republic and its implementation into the companies' strategies is certainly still not on the level of developed Western countries, and much has to be done to improve the situation and to reinforce the perception of ethics in business.

In North-Italian SMEs, many companies have already realized that following ethical principles might be convenient for their financial performance. In this context it would be very interesting to carry out comparative analyses in other countries, European and non, to find out and compare the present state of the perception and implementation of business ethics principles. 


\section{Predictions Of Financial Performance On The Basis Of Non-Financial Aspects}

Our consistent fuzzy model can be further used to predict the economic performance of companies (or other variables). Formally, the predictions are fuzzy queries inputted into the expert system. When making fuzzy queries, we have to determine which variable we want to ask about (dependent variable) and we have to input the values of all the other variables (independent variables). If we cannot estimate these values, we use the value unk (= unknown; this value covers the whole fuzzy interval from low to hig). The fuzzy model will then on the basis of fuzzy similarity generate the requested answer.

\section{Conclusions}

We showed that the application of fuzzy modelling represents a progressive method to predict, on the basis of a set of non-financial variables, financial performance of companies. However, the fuzzy set theory can be a powerful tool for any area where vague, uncertain, ill-structured and sparse data has to be analysed and worked with.

At the same time, we have to be well aware of the limits of the presented method. We cannot expect the algorithms to generate one precise and correct answer, as the data we work with are very vague, incomplete and highly subjective. The quality of the output depends very strongly on the quality of the inputted data.

Equally, no matter how efficient the above-mentioned algorithms may be, they cannot deduce anything, which is not implicitly encoded in them already. In other words, any formal tool can just modify available knowledge items. No formal tool can generate new knowledge. New knowledge item can be gained just from observations. As such, our developed fuzzy model cannot be used for predictions concerning companies in other branches of industry than those included and/or companies in other geografical areas and/or in other periods of time etc. than those described above.

In any case, methods of artificial intelligence, if used reasonably and not with unrealistic expectations, can become progressive tools for studying, analyzing, describing and finding mutual correlations in matters where precise data cannot be obtained.

\section{References}

1. $\quad$ Dohnal, M.: "Fuzzy Flowsheeting", in: The Chemical Engineering J., Vol. 30, p. 71-79, 1985.

2. Dohnal, M., M. Starzak, M. Kerkovsky, J. Dohnalova, J. Vystrcil, R. Koivisto, M.Pokorny, M. Varnis, S. Parsons: "A Fuzzy Upgrading of Integrated Vague Managerial and Engineering Knowledge", in: International Journal of Production Economics, Vol. 32, 1993, p. 209-228.

3. Dubois, D and H. Prade: "Fuzzy Sets in Approximate Reasoning, Part: Inference with Possibility Distributions", Fuzzy Sets and Systems, 40, p. 143-202, 1991.

4. Galassi, Francesco, L.: "A Measure of Culture: Trust and Defection in Southern Italy". p.69-110. In: Cultural Factors in Economic Growth, Springer-Verlag Berlin 2000. 244 p.

5. $\quad$ Kosko, B. and S. Isakei: "Fuzzy Logic's”, in: Scientific American, Vol. 269, No.1, p. 61-67, 1993.

6. Sacconi, L.: "Ethics, Corporate Culture and Economic Modelling", In P. Koslowski (Ed.): Contemporary Economic Ethics and Business Ethics, Springer-Verlag Berlin 2000. p. 80-111.

7. Vaija, P., M. Jarvelainen, M. Dohnal: "Failure Diagnosis of Complex Systems by a Network of Expert Basis", Reliability Engineering J., 16, p. 237-251, 1986.

8. Zimmermann, H.J.: Fuzzy Sets Theory and Its Applications, Kluwer-Nijhoff, Amsterdam 1986. 
Notes 